\title{
Insight into metabolic pathways of the potential biofuel producer, Paenibacillus polymyxa ICGEB2008
}

\author{
Nidhi Adlakha ${ }^{1 \dagger}$, Thomas Pfau $2,3{ }^{\dagger}$, Oliver Ebenhöh ${ }^{2,4^{*}}$ and Syed Shams Yazdani ${ }^{* *}$
}

\begin{abstract}
Background: Paenibacillus polymyxa is a facultative anaerobe known for production of hydrolytic enzymes and various important biofuel molecules. Despite its wide industrial use and the availability of its genome sequence, very little is known about metabolic pathways operative in the Paenibacillus system. Here, we report metabolic insights of an insect gut symbiont, Paenibacillus polymyxa ICGEB2008, and reveal pathways playing an important role in the production of 2,3-butanediol and ethanol.

Result: We developed a metabolic network model of P. polymyxa ICGEB2008 with 133 metabolites and 158 reactions. Flux balance analysis was employed to investigate the importance of redox balance in ICGEB2008. This led to the detection of the Bifid shunt, a pathway previously not described in Paenibacillus, which can uncouple the production of ATP from the generation of reducing equivalents. Using a combined experimental and modeling approach, we further studied pathways involved in 2,3-butanediol and ethanol production and also demonstrated the production of hydrogen by the organism. We could further show that the nitrogen source is critical for metabolite production by Paenibacillus, and correctly quantify the influence on the by-product metabolite profile of ICGEB2008. Both simulations and experiments showed that metabolic flux is diverted from ethanol to acetate production when an oxidized nitrogen source is utilized.
\end{abstract}

Conclusion: We have created a predictive model of the central carbon metabolism of P. polymyxa ICGEB2008 and could show the presence of the Bifid shunt and explain its role in ICGEB2008. An in-depth study has been performed to understand the metabolic pathways involved in ethanol, 2,3-butanediol and hydrogen production, which can be utilized as a basis for further metabolic engineering efforts to improve the efficiency of biofuel production by this $P$. polymyxa strain.

Keywords: Paenibacillus polymyxa, Metabolic modeling, Ethanol, 2,3-Butanediol, Metabolic engineering, Redox metabolism

\section{Background}

The ecological roles of Paenibacillus polymyxa are highly diverse and have been described in the literature for various important symbiotic relationships. It was found to be associated with plants where it helps in $\mathrm{N}_{2}$ fixation

\footnotetext{
*Correspondence: oliver.ebenhoeh@hhu.de; shams@icgeb.res.in ${ }^{\dagger}$ Nidhi Adlakha and Thomas Pfau contributed equally to this work 1 Synthetic Biology and Biofuels Group, International Centre for Genetic Engineering and Biotechnology, Aruna Asaf Ali Marg, New Delhi, India ${ }^{2}$ Institute of Complex Systems and Mathematical Biology, University of Aberdeen, Aberdeen, UK

Full list of author information is available at the end of the article
}

[1] and secreting growth hormones [2], and it was also observed in the gut of insects where it helps the insects to digest their food by secreting various enzymes capable of hydrolyzing organic materials [3]. P. polymyxa is a non-pathogenic strain for the production of industrially valuable 2,3-butanediol [4], which is a precursor for various fuels and petrochemical products. So far genome sequences of 14 P. polymyxa strains have been deposited at NCBI. However, the genome annotation is far from complete and, to our knowledge, metabolic reconstructions have not been performed for any Paenibacillus sp. 
The investigation of metabolic pathways allows indepth insight into the molecular mechanisms of a particular organism. The improved understanding of the architecture of cellular metabolism and the enormous amount of genomic data available today can help draw the entire metabolic map of a cell and redesign it by rational and directed metabolic engineering. The detailed biosynthetic pathways have been established for several model microorganisms such as Escherichia coli $[5,6]$ and Saccharomyces cerevisiae [7, 8]. But, despite wide industrial use and availability of the genome sequence for P. polymyxa, very little is known about its metabolism [9, 10].

In this report, the metabolic capabilities of Paenibacillus sp. ICGEB2008 (referred to as ICGEB2008) [11] have been studied. This strain was isolated from the gut of a cotton bollworm and was shown to produce a number of cellulolytic enzymes $[12,13]$. The strain also showed the ability of producing high yields of 2,3-butanediol [14]. In combination, these metabolic capabilities make this strain an interesting candidate for biotechnological purposes, which include the conversion of biomass to combustible fuels or valuable chemicals. To interpret the results obtained from sequence analysis and to obtain new insights into the biochemical capabilities of this strain, we reconstructed a metabolic network model of the carbon metabolism of ICGEB2008 by integrating genomic and biochemical data, resulting in a stoichiometric model connecting 133 metabolites by 158 reactions. Employing flux balance analysis (FBA) [15, 16], we could support the putative annotation of a formate hydrogen lyase and enzymes of the Bifid shunt. The pathways involved specifically in ethanol and 2,3-butanediol production have been studied in detail, generating an understanding that will be useful for engineering the Paenibacillus system for improved biofuel production. We simulated growth on different nitrogen sources and predicted the resulting composition of by-products excreted into the medium. For experimental confirmation, we performed growth experiments, which supported the simulated by-product profiles. In combination, the model and data presented here can serve as a basis for further metabolic engineering and provide an improved insight into the metabolic capabilities of ICGEB2008.

\section{Results and discussion}

\section{Pathways for fermentative products}

Several reports highlight $P$. polymyxa as a non-pathogenic, non-obligatory host for 2,3-butanediol production $[4,17]$. Our experiments confirmed this for ICGEB2008 showing a maximal yield of $0.32 \mathrm{~g}$ 2,3-butanediol per $\mathrm{g}$ of glucose ( 0.49 mM 2,3-butanediol/mM glucose) (Fig. 1) [14]. In addition, we also observed ethanol secreted with

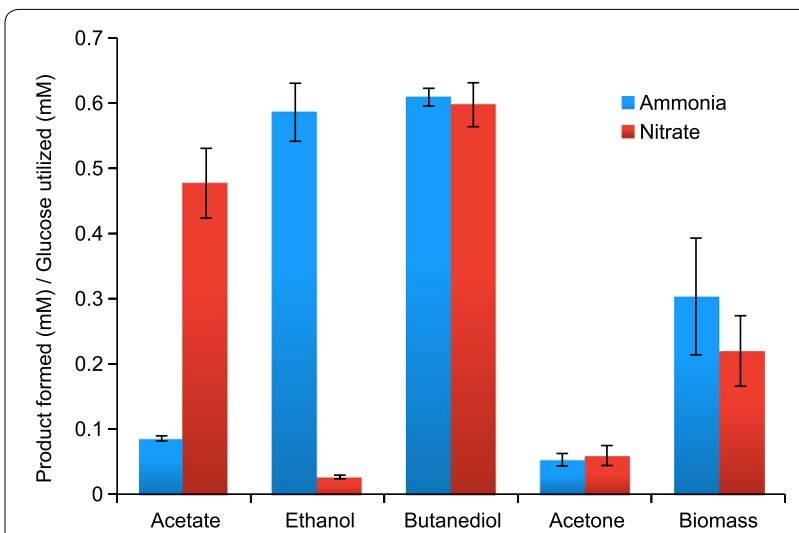

Fig. 1 Product profile of P. polymyxa ICGEB2008 under anaerobic condition of growth with two different nitrogen sources. The experiments were performed in triplicate by growing the culture in $50 \mathrm{ml}$ medium for $24 \mathrm{~h}$ and analyzing the extracellular metabolites via HPLC. The results represent average and standard deviation of data from three biological replicates

a yield of $0.18 \mathrm{~g}$ per g glucose $(\sim 0.7 \mathrm{mM}$ ethanol $/ \mathrm{mM}$ glucose), besides small amounts of acetic acid, acetone and lactic acid. Sequence analysis confirmed the presence of genes encoding enzymes involved in 2,3-butanediol (als, aldB, bdh), ethanol (adh, ald) and acetic acid (pta, ack) production (Additional file 1: Figure S1, accession number available in Additional file 2). Further, enzymes involved in acetone production were annotated. Interestingly, a gene encoding fructose-6-phosphate phosphoketolase (F6PK) was annotated, which is responsible for a glycolytic bypass pathway called Bifid Shunt. We experimentally validated the functionality of the Bifid shunt by confirming the F6PK activity as $43 \mathrm{nmol} / \mathrm{min} / \mathrm{mg}$ cellular protein in ICGEB2008 (Additional file 1: Figure S2).

The "Bifid shunt", or glucose catabolism through the fructose 6-phosphate phosphoketolase (EC 4.1.2.22), generates acetyl phosphate and erythrose 4-phosphate. The final products of the fermentation route are formed by the sequential action of the enzymes: transaldolase (EC 2.2.1.2), transketolase (EC 2.2.1.1.) and xylulose 5-phosphate phosphoketolase (EC 4.1.2.9) which generates glyceraldehyde 3-phosphate, that enters the Embden-Meyerhof-Parnas pathway [18], and acetyl phosphate, which is converted to the final product acetate. Conversion of glucose to acetate through the standard glycolytic pathway yields 4 molecules of ATP and 2 molecules of NADH per molecule glucose (Eq. 1), which also corresponds to the maximal ATP yield (2/3 ATP per carbon, see Table 1). However, the Bifid shunt (Fig. 2) allows to bypass glycolysis and to convert $100 \%$ of the carbons in glucose into acetate (value 1 in Table 1). This pathway results in a lower yield of ATP (2 ATP per glucose), but does not produce reductants (Eq. 2). 
Table 1 Conversion and production yields on a per carbon basis

\begin{tabular}{lllll}
\hline $\begin{array}{l}\text { Substrate } \Rightarrow \\
\text { Product } \Downarrow\end{array}$ & Cellobiose & Glucose & Glycerol & Xylose \\
\hline Acetate & 1.0 & 1.0 & $1.0(0.67)$ & 1.0 \\
Acetone & 0.75 & 0.75 & $0.71(0.2)$ & 0.75 \\
Butanediol & 0.73 & 0.73 & $0.83(0.67)$ & 0.73 \\
Ethanol & 0.67 & 0.67 & 0.78 & 0.67 \\
Energy (ATP) & $0.75(0.5)$ & $0.66(0.42)$ & $0.66(0.11)$ & $0.66(0.5)$ \\
Biomass & $0.72(0.68)$ & $0.64(0.59)$ & $0.63(0.17)$ & $0.64(0.59)$
\end{tabular}

Energy in ATP refers to the possible hydrolysis of ATP into ADP and Pi per consumed carbon of the given substrate. To quantify the effect of surplus reductants on the results, we performed the simulations without the FHL reaction. The corresponding numbers are given in parentheses

$$
\begin{aligned}
& 1 \text { Glucose } \rightarrow 2 \text { Acetate }+4 \text { ATP }+2 \mathrm{CO}_{2} \\
& +2 \mathrm{NADH}(\text { Glycolytic route })
\end{aligned}
$$

\section{Glucose $\rightarrow 3$ Acetate + 2ATP (Bifid Shunt)}

The simultaneous presence of these two pathways introduces a degree of flexibility into metabolism by allowing uncoupling of the production of energy and redox equivalents. As discussed below, this regulatory potential is critically important for anaerobic growth, where electron sinks are not abundant.

\section{Broad substrate specificity}

Due to its capability of producing a number of enzymes hydrolyzing diverse polymeric, oligomeric or dimeric carbohydrates [12, 13], ICGEB2008 is able to grow on a broad spectrum of substrates. This is supported by genome sequence analysis revealing the presence of monosaccharide and disaccharide transporters, allowing utilization of the breakdown products (Table 2). This was validated experimentally by growing ICGEB2008 on different carbon sources under anaerobic conditions (Table 2). Using constraint-based modeling, we calculated maximal carbon and energy yields for various substrates. For a variety of natural carbon sources, the maximal yields of potential products (in carbon per carbon) and ATP (in molecules ATP per carbon) are presented in Table 1. The highest ATP yield per carbon is observed for cellobiose, which is slightly higher than for glucose because of the phosphorylative degradation via cellobiose phosphorylase (CEP) [19].

\section{Reconstruction and analysis of a metabolic model}

Mathematical models are useful to understand the biochemical capabilities of an organism and to make predictions about the impact of genetic and/or environmental perturbations on metabolic fluxes and growth. Clearly, such understanding supports the development of targeted strategies to improve the yield of the valuable product 2,3-butanediol in biotechnological applications. Therefore, we constructed a stoichiometric model of ICGEB2008 focussing on carbon metabolism. From the genome sequence and the MetaCyc database [20], we assembled a network model containing 133 metabolites and 158 reactions (for details see "Methods"). A representation of the central metabolic routes is given in Additional file 1: Figure S1. To simulate growth, we included in the model reactions describing the conversion of precursors from primary metabolism (such as pyruvate, succinate and ammonia) into the biomass components for DNA/RNA (i.e., single nucleotides), proteins (i.e., connected chains of amino acids), lipids (i.e., glycerolipids) and cell wall (i.e., peptidoglycans). These processes are described as 'lumped' reactions, in the sense that they represent a series of biochemical processes instead of single enzymatic reactions. In the following, 'biomass' is measured in the unit of incorporated carbon. We confirmed that the model is capable of producing cellular precursors in experimentally determined ratios from minimal medium on various carbon sources (glucose, xylose, cellobiose and glycerol). We used the experimentally observed cellular composition as a constraint to further analyze the model (Table 3 ).

\section{Limitations of anaerobic growth}

The production of 2,3-butanediol and ethanol was observed majorly under anaerobic conditions (Additional file 1: Figure S3). So, we imposed additional constraints to mimic anaerobic lifestyle in ICGEB2008. The most important constraint is the lack of oxygen as electron acceptor. This poses severe limitations on the overall metabolism, requiring alternative electron sinks. Most importantly, in the absence of oxygen, the strain is not able to produce ATP through oxidative phosphorylation, which imposes a major limitation for anaerobic growth. For example, whereas maximal carbon yields using sugars as substrates are independent of the presence of a redox dissipating mechanism, the biomass yield on glycerol, which is more reduced than sugars, is severely reduced under anaerobic conditions and, therefore, requires an additional oxidation (see Table 1 ). We investigated the most efficient anaerobic pathways in the model to produce ATP if glucose is the only carbon source. The highest yield of ATP can be obtained with a complete conversion of glucose to acetate, where 4 molecules of ATP can be produced per molecule of glucose. This includes an additional molecule of ATP after fermentation of glucose to pyruvate via phosphate acetyl transferase (PTA) and acetate kinase (ACK). However, only 


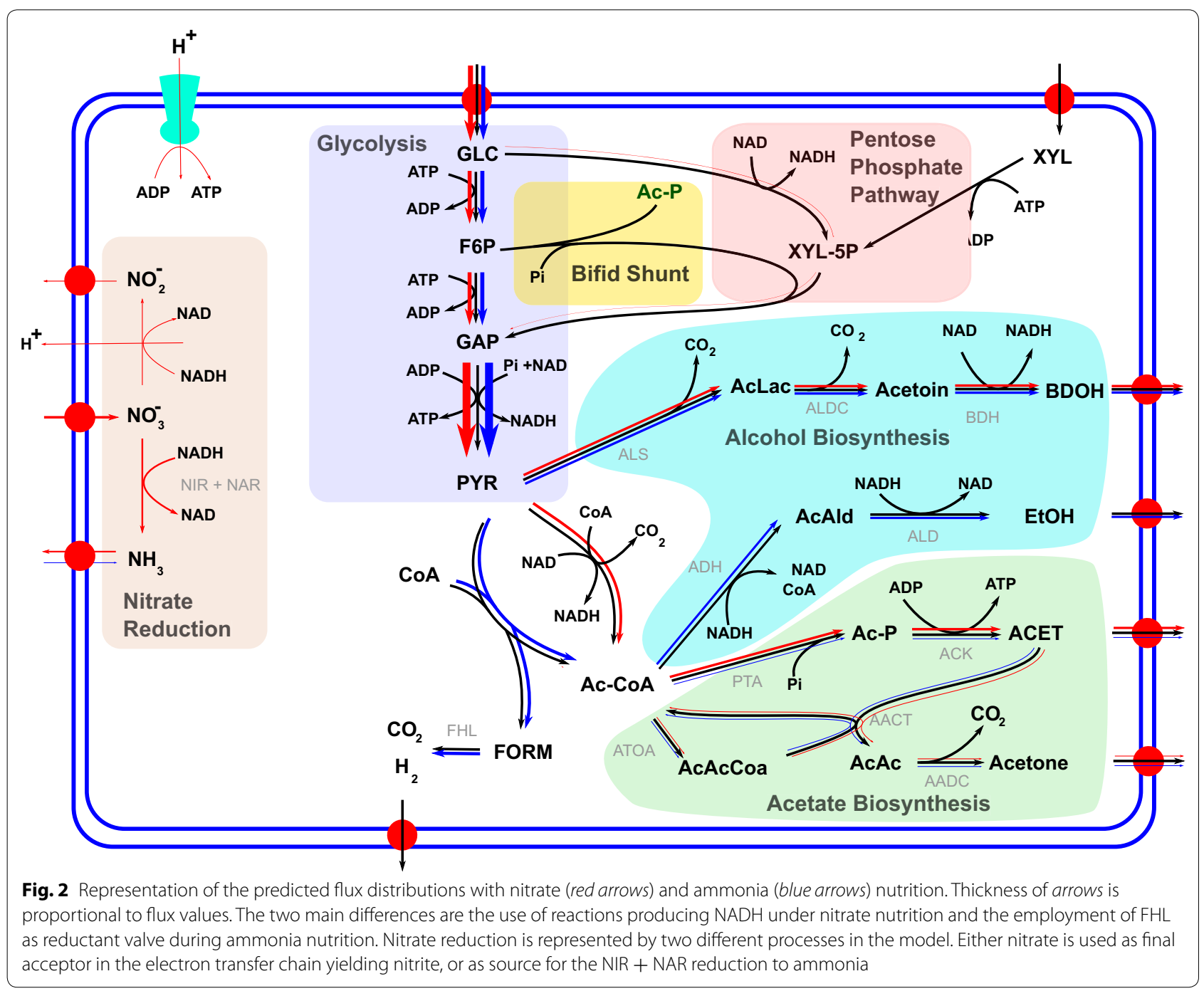

Table 2 Sugar transporters annotated in the genome of $P$. polymyxa ICGEB2008

\begin{tabular}{|c|c|c|c|}
\hline Sugar & Transporter & Protein ID & Substrate utilized $^{\mathrm{a}}$ \\
\hline Glucose & PTS system $2 \mathrm{C}$ glucose-specific components & WP_017426741.1,WP_016818550.1 & $5.0 \mathrm{~g} / \mathrm{l}$ \\
\hline Xylose & Xylose $\mathrm{ABC}$ transporter $2 \mathrm{C}$ permease component & $\begin{array}{l}\text { WP_017426799.1,WP_017426800.1,WP_017426801.1, } \\
\text { WP_017427837.1,WP_017428351.1 }\end{array}$ & $3.78 / 1$ \\
\hline Sucrose & PTS system 2C sucrose-specific component & WP_017426394.1,WP_017426681.1,WP_016820208.1 & $4.52 / 1$ \\
\hline Maltose & $\begin{array}{l}\text { Maltose/maltodextrin } \mathrm{ABC} \text { transporter } 2 \mathrm{C} \text { permease } \\
\text { protein MalF }\end{array}$ & WP_017427403.1 & $4.45 \mathrm{~g} / \mathrm{l}$ \\
\hline Lactose & Lactose transport system (lactose-binding protein) & WP_017425625.1 & $4.16 \mathrm{~g} / \mathrm{l}$ \\
\hline Cellobiose & PTS system $2 \mathrm{C}$ cellobiose-specific component & WP_017428673.1,WP_017428674.1,WP_016819955.1 & $5.0 \mathrm{~g} / \mathrm{l}$ \\
\hline Xylobiose & Xyloside transporter XynT & WP_017427857.1,WP_017426938.1 & $1.49 \mathrm{~g} / \mathrm{l}$ \\
\hline Polysaccharide & $\begin{array}{l}\text { Polysaccharide } A B C \text { transporter substrate-binding pro- } \\
\text { tein, polysaccharide } A B C \text { transporter permease }\end{array}$ & WP_017425534.1,WP_016822415.1 & $1.18 \mathrm{~g} / \mathrm{l}($ Starch) \\
\hline
\end{tabular}

\footnotetext{
${ }^{a}$ HPLC measurement for substrate unutilized when ICGEB2008 was grown for $48 \mathrm{~h}$ in minimal media containing $5 \mathrm{~g} / \mathrm{l}$ of respective substrate
} 
Table 3 Biomass composition for P. polymyxa ICGEB2008

\begin{tabular}{ll}
\hline Cellular component & Composition (\%) \\
\hline Protein & 48.3 \\
DNA/RNA & 5.55 \\
Lipids & 6.8 \\
Cell wall & 20 \\
\hline
\end{tabular}

small amounts of acetate are experimentally observed as by-product (Fig. 1). This can readily be explained by the strong pH-dependent growth of ICGEB2008 [14], suggesting that the organism avoids the production of excess acidic compounds. We, therefore, imposed an additional constraint on the model and restricted the export of acidic compounds to a total representing the experimentally observed amounts $(0.123 \mathrm{mM} / \mathrm{mM}$ glucose based on growth on minimal media). Also, the production of acetone allows for a larger fraction of the carbon to be redirected through acetate synthesis, where additional ATP can be produced. While excretion of acetate would yield even higher ATP amounts, it would simultaneously give rise to higher acid export, which is restricted in the model. Acetone export can circumvent this issue while still allowing the generation of an additional ATP after fermentation of glucose to pyruvate. However, only small amounts of acetone were observed in our experiments. It is likely that the limiting reaction for acetone production is the one catalyzed by acetoacetate CoA transferase (ATOAD), because this enzyme is inhibited by physiological levels of its own product acetone and butanol [21]. The latter is particularly interesting, as 2,3-butanediol could have a similar effect, thus reducing the activity to the observed amounts.

\section{Formate-hydrogen lyase as a redox valve}

ATP is required for both growth-related and maintenance processes. It is relatively easy to estimate growthrelated ATP requirement, but the ATP requirement for non-growth-related processes is far more difficult to assess. One possibility is to systematically vary the flux through an ATP consuming (ATPase) reaction, which mimics the additional ATP maintenance demand, and predict growth and by-product formation and comparing the predicted values with experimentally determined quantities. In Fig. 3, the result of such an ATP requirement scan is depicted. Best agreement with experimentally observed growth is found for an ATPase flux of 6.48 ATP/biomass carbon (Fig. 3), with ethanol as the only predicted by-product. This by-product profile is clearly not in agreement with the experimentally observed mixture of various substances. The model predicts a high ethanol production, mainly because it utilizes ethanol

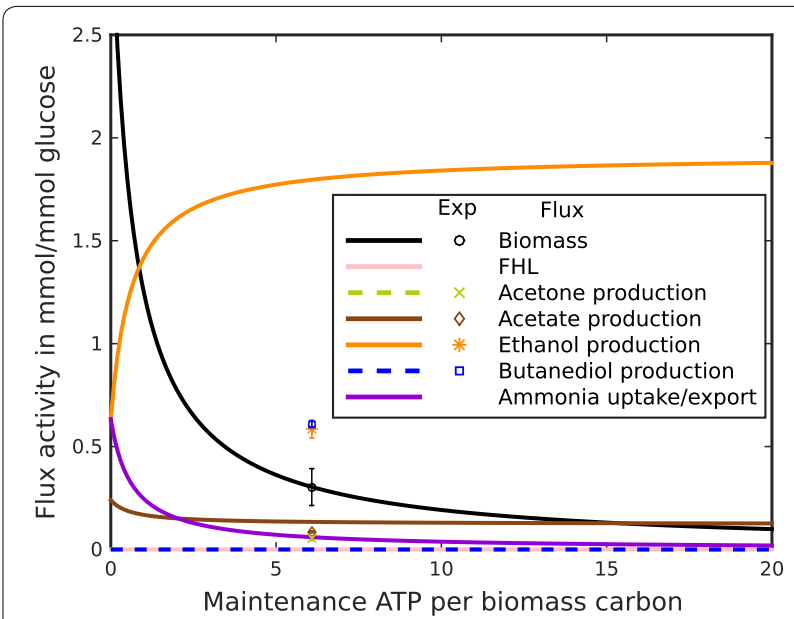

Fig. 3 Scan over a range of maintenance ATP required per biomass carbon for the model without formate hydrogen lyase (FHL) activity. One unit of biomass refers to one carbon of newly produced P. polymyxa. Markers show the experimental values for biomass, ethanol and 2,3-butanediol production

excretion as an efficient way to remove excess reductants from the system. To resolve the discrepancy between model and experiment, we hypothesized the presence of an alternative mode of reductant dissipation. To find the alternate pathway, we analyzed the genome sequence of ICGEB2008 and found a gene coding for a formatehydrogen lyase (FHL), which utilizes NADH and produces hydrogen. This hydrogenase uses protons as final electron acceptors and can act as a mode to dissipate excess NADH. The hypothesis was supported by hydrogen production in other Paenibacillus strains [22], which we could also confirm for ICGEB2008 by GC analysis (Additional file 1: Figure S4).

Incorporation of this enzyme into the model improved the prediction of by-products considerably (Additional file 1: Figure S5). However, the production of ethanol was still slightly overestimated and the production of 2,3-butanediol slightly underestimated. Thus, the model predicted a more reduced total by-product combination than experimentally observed. To quantify the discrepancy between the observed and predicted requirement of reductants, we included a reductant removing reaction in the model and systematically varied the corresponding flux (Fig. 4a) in analogy to the method described above to estimate maintenance ATP requirements. This analysis revealed a best fit to the observed growth and by-product ratios for an additional consumption of reductant of about 0.7 NADH per biomass carbon (Fig. 4b). To identify the additional electron sink, which could explain the origin of this discrepancy, we analyzed the model prediction for the formation of $\mathrm{CO}_{2}$, a byproduct with 

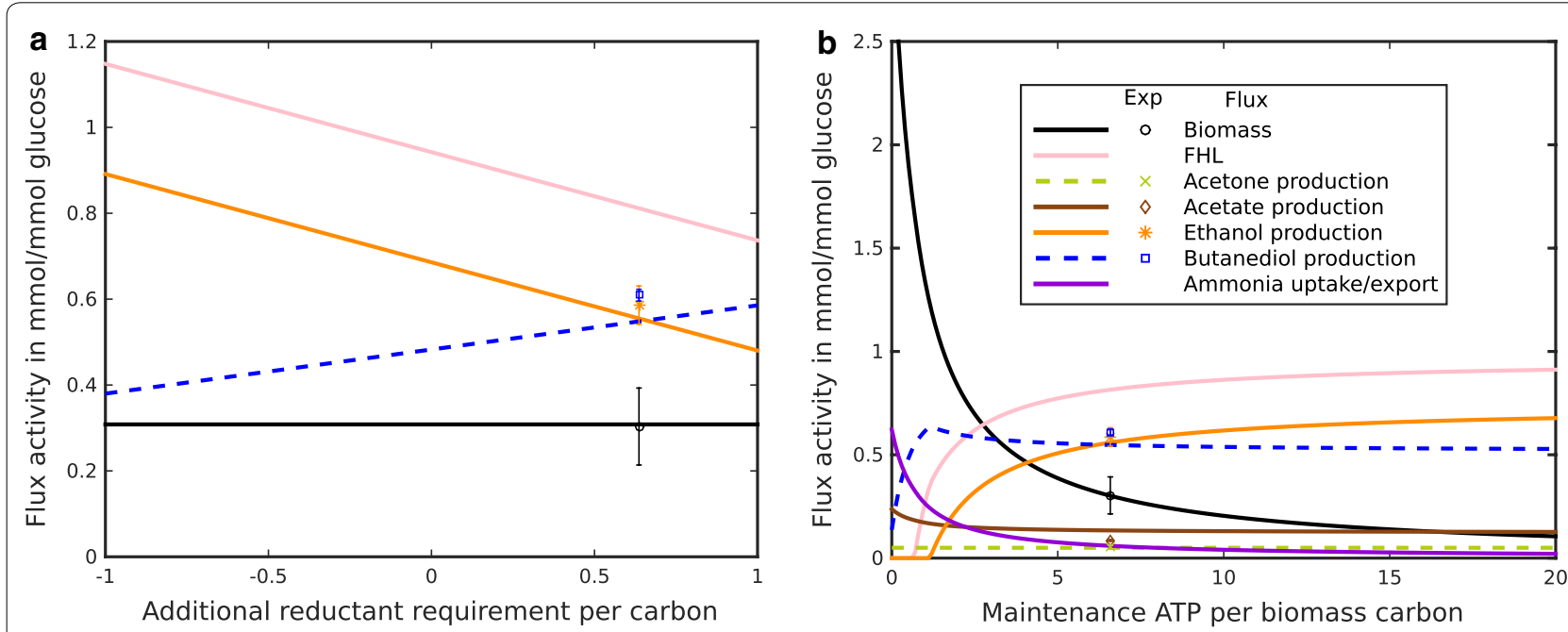

Fig. 4 a Scan over the strength of an additional electron sink to identify the amount of additional reduction required to predict the experimentally observed by-product formation. Predicted ratio of ethanol/butanediol shifts towards 2,3-butanediol in response to oxidized medium. Positive values indicate additional flux through the DEHOG reaction and thus either an increased demand of reductant or a more reduced biomass. Markers show the experimental values for biomass, ethanol and 2,3-butanediol production. The simulated outputs fit well to these when using FHL and an adjusted redox demand of $+0.7 /$ biomass carbon (see text). b Scan over a range of maintenance ATP required per biomass carbon as in Fig. 3 , but with $\mathrm{FHL}$ and an additional reductant requirement of $+0.7 /$ biomass carbon, as determined by Fig. 4a)

extreme oxidation state, which was not experimentally measured. The predicted value of $\mathrm{CO}_{2}$ formation under the assumption of biomass maximization was $1.96 \mathrm{mM} /$ $\mathrm{mM}$ glucose. To study whether this value was potentially overestimated by the model, we systematically fixed the $\mathrm{CO}_{2}$ formation to values between 1.5 and $2 \mathrm{mM} / \mathrm{mM}$ glucose (Additional file 1: Figure S6). Apparently, with lower $\mathrm{CO}_{2}$ production the by-products become less reduced. The best fit to the experimentally observed values was obtained for a value of $1.83 \mathrm{mM} \mathrm{CO} / \mathrm{mM}$ glucose. This fit is of the same quality as that obtained by assuming an additional electron sink (Fig. 4b). We, therefore, conclude that the discrepancy between model prediction and observed by-product formation (Additional file 1: Figure S5) results from an overestimation of $\mathrm{CO}_{2}$ production and that this overestimation can be corrected by assuming an additional electron sink.

\section{Importance of $\mathrm{N}$-source and altering the redox balance}

Given the constraints on anaerobic metabolism by the unavailability of molecular oxygen for the dissipation of reductants, it appears plausible that the redox state of the nutrients will influence by-product metabolite production of ICGEB2008. Since nitrate can be used as a terminal electron acceptor [23], we investigated the effect of supplying nitrate instead of ammonium as nitrogen source. It is expected that under growth on nitrate, reductants are no longer in excess, but rather required for nitrate reduction. The model consequently predicted a strong increase in acetate production and a decrease in ethanol production (Fig. 5). To test this prediction experimentally, we grew ICGEB2008 in a medium in which ammonia was replaced by nitrate and found that the model correctly predicted that 2,3-butanediol and acetate are the only major expected by-products (Fig. 1). The model further suggested that nitrate is used as final electron acceptor and is reduced to ammonia, which is exported. This hypothesis was supported by the genome analysis, which revealed two corresponding nitrate reductase gene clusters in the genome of ICGEB2008 (Additional file 1: Figure S7). For experimental confirmation, we measured the ammonium content in the extracellular medium and found that after $48 \mathrm{~h}$ approximately a third of the initially applied nitrate had been reduced to ammonia (Additional file 1: Figure S8). We further observed the presence of nitrite in the final solution and adapted the model accordingly. Constraining nitrite and ammonia export to the experimentally observed values resulted in a predicted by-product composition closely reflecting the experimentally observed values (Fig. 5). The calculated flux distributions for the best fits for nitrate and ammonium nutrition are depicted in Fig. 2. The main differences arise from a flux through reductant producing pathways under nitrate nutrition, such as the use of pyruvate dehydrogenase $(\mathrm{PDH})$ instead of pyruvate-formate lyase (PFL). An additional effect is the inactivation of the Bifid shunt. The availability of nitrate as terminal electron acceptor allows removing excess reductants and, 


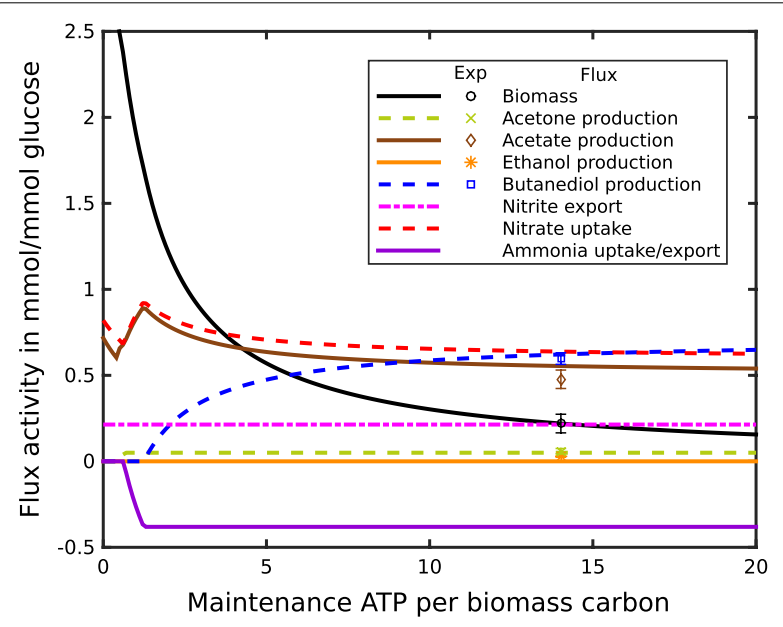

Fig. 5 ATP requirement scan with nitrate as nitrogen source. The model predicted ammonium production, which was experimentally confirmed for growth of P. polymyxa ICGEB2008 on nitrate. Constraining the total ammonium production in the model to experimentally observed values, the model predicts a decrease in ethanol production to zero and an increase in acetate formation, which is in good qualitative agreement with experimental data. The simulations suggest that the maintenance ATP requirement per biomass almost doubles on nitrate when compared to ammonium nutrition therefore, the standard glycolytic pathway is operable. Thus, in contrast to growth on ammonium, the "reductant free" ATP that can be regenerated by the Bifid shunt is no longer necessary.

\section{Conclusions}

The diverse metabolic capabilities of Paenibacillus polymyxa, ranging from the degradation of diverse organic compounds in the gut of insects to the production of industrially relevant biomolecules like 2,3-butanediol, make this organism a particularly interesting candidate for a detailed investigation of its metabolic potential. They also highlight that $P$. polymyxa is of considerable interest for potential biotechnological applications. Here, we demonstrated the capability of the ICGEB2008 strain to produce biofuels, in particular ethanol and 2,3-butanediol. To understand the metabolic pathways operative in ICGEB2008 and to develop strategies to stimulate production of biofuels in this strain, we have built a metabolic model and applied flux balance analysis (FBA) to explain and predict the by-product formation under different growth conditions. Our model successfully predicted the influence of the redox balance in ICGEB2008. Using a combinatorial experimental and modeling approach, we have shown the influence of the redox state of the nitrogen source on by-product yields and composition. We predicted a diversion of metabolic flux from ethanol to acetate in more oxidizing environments, which we experimentally confirmed by growing ICGEB2008 on nitrate. The model further supported the existence of a formate hydrogen lyase as redox valve. By the detection of hydrogen in the overhead, we could further support this finding experimentally. This finding could serve as a starting point for further development of the organism as a potential hydrogen producer as an additional useable by-product. We have also elucidated the presence of the Bifid shunt in the bacterium as a bypass for the glycolytic pathway, which is active under anaerobic growth without the presence of an alternative electron acceptor such as nitrate.

In summary, we have developed a metabolic model for the industrially relevant strain, Paenibacillus polymyxa ICGEB2008 and investigated the metabolic pathways operative in this strain. We have demonstrated the potential of metabolic modeling to simulate the capabilities of ICGEB2008 and elucidated the Bifid shunt in P. polymyxa ICGEB2008. In addition, we have shown that the redox state of the nitrogen source is critical for the by-product profile. The present study can serve as a basis for further metabolic engineering efforts to improve the efficiency of biofuel production by this $P$. polymyxa strain.

\section{Methods}

\section{Culture media and cultivation conditions}

For biomass composition and product analysis, ICGEB2008 was grown in minimal medium [24] containing $5 \mathrm{~g} / \mathrm{l}$ glucose. The growth in nitrate medium was obtained by replacing ammonium chloride with an equimolar quantity of sodium nitrate. The effect of acetate on ICGEB2008 metabolism was studied by adding the specified amount of acetate after $\mathrm{OD}_{600}$ reached 0.8 and the culture was grown further for $48 \mathrm{~h}$. The products secreted were quantified using HPLC. Substrate specificity was established by growing ICGEB2008 anaerobically in $125 \mathrm{ml}$ serum bottles containing media with $2.5 \mathrm{~g} / \mathrm{l}$ of different carbon sources. The carbon source utilization was estimated using HPLC. The values obtained for cell biomass, substrate, utilization and product synthesis were used for calculation of biomass and product yields ( $\mathrm{mmol} / \mathrm{mmol}$ substrate). For calculating biomass yield, a molecular formula $\mathrm{CH}_{1.9} \mathrm{O}_{0.5} \mathrm{~N}_{0.2}$ of cells was used with an average molecular weight of 24.7 [25].

\section{Cell composition analysis}

Different components of cells were analyzed for their composition [26, 27]. Cell wall was extracted by repeated washing of a known mass of ground lyophilized tissue with a mixture of phenol, acetic acid, and water in the ratio 2:1:2 [26]. The remaining insoluble material was washed with distilled water, freeze dried, and weighed as cell wall component. Lipids were extracted from a known 
mass of ground-lyophilized tissue using hexane and isopropanol according to an established protocol [27]. Solvent was removed by gentle heating, and residues were weighed as lipid component. Soluble protein extracted with phosphate-buffered saline was quantified against BSA standard using the BCA protein assay kit (Bio-Rad). Nucleic acids were extracted from lyophilized tissue using standard methods (i.e., for RNA, TRIzol extraction followed by DNase treatment; for DNA, phenol/chloroform/isoamyl alcohol extraction followed by RNase treatment) and quantified spectrophotometrically.

\section{Genome annotation and subsystem analysis}

The initial annotation of coding sequences of ICGEB2008 was achieved using the automated server RAST (http:// rast.nmpdr.org/) [28], which is available at NCBI website with Reference Sequence No. NZ_AMQU00000000.1. While constructing the metabolic pathways, the missing link in the network was identified by Reverse Blast Hit (RBH) strategy with BLAST threshold at $1 \mathrm{e}^{-05}$ and their annotations have been provided in the Additional file 2 . Gene clusters were analyzed using img/er server (https:// img.jgi.doe.gov).

\section{Analytical assays}

Ammonium ion production was estimated as follows. To $1.5 \mathrm{ml}$ culture supernatant, $50 \mu \mathrm{l}$ manganous salt solution, $1 \mathrm{ml}$ alkaline phenol reagent and $0.5 \mathrm{ml}$ hypochlorite solution were added. The reaction mix was boiled for $5 \mathrm{~min}$ and color development was monitored at $625 \mathrm{~nm}$ [29]. Residual nitrate estimation was done using a modification of the method described by Middleton [30]. To $0.5 \mathrm{ml}$ of culture filtrate, $5 \mathrm{ml}$ of $0.55 \% \mathrm{Ca}\left(\mathrm{CH}_{3} \mathrm{COO}\right)_{2} \cdot \mathrm{H}_{2} \mathrm{O}$ in $4 \%$ ammonia, $0.1 \mathrm{ml}$ of $1 \% \mathrm{MnSO}_{4} \cdot 4 \mathrm{H}_{2} \mathrm{O}$ in $5 \%$ acetic acid, and about $0.1 \mathrm{~g}$ of finely powdered zinc were added. This mixture was shaken vigorously for $1 \mathrm{~min}$ and filtered; $2 \mathrm{ml}$ of the filtrate was placed on ice and $0.5 \mathrm{ml}$ of $1 \%$ sulfanilamide in $5 \mathrm{~N} \mathrm{HCl}$ was added. The sample was incubated on ice for $15 \mathrm{~min}$ followed by the addition of $0.5 \mathrm{ml}$ of $0.02 \%$ $\mathrm{N}$-(1-naphthyl)-ethylenediamine solution and incubation at room temperature for $30 \mathrm{~min}$. After incubation, $2 \mathrm{ml}$ of water was added and absorbance was measured at $540 \mathrm{~nm}$ in spectrophotometer.

Fructose-6-phosphate phosphoketolase was assayed based on Tannock's protocol [31] as follows. The cells were harvested by centrifugation after 24-h cultivation in Scheper's minimal medium. The bacterial cells were washed using $10 \mathrm{ml}$ of $0.05 \mathrm{M}$ phosphate buffer and finally suspended in $1 \mathrm{ml}$ of phosphate buffer containing $3 \mathrm{mg}$ lysozyme. The cells were then lysed by sonication used for the assay. The cell lysate $(100 \mu \mathrm{l})$, sodium fluoride-iodoacetic acid solution $(24 \mu \mathrm{l}$ of $6 \mathrm{mg} / \mathrm{ml})$ and fructose-6-phosphate $(24 \mu \mathrm{l}$ of $12 \mathrm{mg} / \mathrm{ml})$ were added to the test wells and the reaction was incubated at $37^{\circ} \mathrm{C}$ for $30 \mathrm{~min}$. The fructose-6-phosphate was not added in the negative control well. The reaction was stopped by adding $150 \mu \mathrm{l}$ of hydroxylamine solution, $100 \mu \mathrm{l}$ of trichloroacetic acid solution and $100 \mu \mathrm{l}$ of $4 \mathrm{M}$ hydrochloric acid. A reddish-violet color was obtained after addition of $100 \mu \mathrm{l}$ of ferric chloride solution indicating fructose6-phosphate phosphoketolase activity, which was estimated spectrophotometrically at $505 \mathrm{~nm}$.

Hydrogen was estimated by growing cells anaerobically in minimal media in the sealed serum bottle for $36 \mathrm{~h}$ and headspace gases were analyzed by GC (Carboxen-1010 Plot column in Perkin Elmer's Clarus 500GC) for hydrogen estimation.

\section{Model construction and curation}

The metabolic model of ICGEB2008 [11] was reconstructed based on an initial annotation of the central carbon metabolism by RAST [28] and protein name and EC number matching in MetaCyc. To reduce complexity, batch reactions for amino acid, nucleotide and lipid biosynthesis were introduced based on MetaCyc Pathways. ScrumPy [32] was used as modeling tool and to perform flux balance analysis with a dual objective of biomass optimization followed by flux minimization. The solver employed was CPLEX 12.6 with an interface for ScrumPy. To obtain a comprehensive and organism specific network, we extracted all reactions catalyzed by enzymes from both the central carbon metabolism and fermentation subcategories of RAST's carbohydrate metabolism group. For these enzymes, we extracted the EC numbers and retrieved the corresponding reactions from MetaCyc using ScrumPy as modeling tool. The retrieved reactions were manually filtered as many retrieved EC numbers link to unspecific reactions (e.g., aldehyde dehydrogenase). In MetaCyc these EC numbers can match very specialized reactions which do not have their own EC number and are only present in specialized organisms. In addition, we removed reactions which produce compounds that were not observed in Paenibacillus and were only present in single reactions.

We introduced batch reactions for biosynthesis of the 20 proteinogenic amino acids and all nucleotides (four ribonucleic acids and four deoxyribonucleic acids). Finally, we added two batch reactions for lipid biosynthesis-one forming palmitic acid and another to convert three palmitic acids and glycerol-phosphate into one lipid. We chose this simplification over the actual lipid metabolism because the general requirements for energy and reductant are similar enough for our study between the different lipids. To create these batch reactions, the biosynthesis pathways from MetaCyc were 
combined into one reaction by adding up all substrates and products.

The current model is restricted to anaerobic conditions and, therefore, works under a strict redox balance. However, we have included a batch electron transfer chain using nitrate as substrate. This reaction mainly converts ADP, Pi, nitrate and $\mathrm{NADH}$ to $\mathrm{NAD}^{+}$, water, nitrite and ATP.

\section{Network reconstruction and modeling}

Metabolic models allow us to investigate the metabolism of the modelled organisms. A robust method often used in this kind of studies is flux balance analysis (FBA) [15]. The huge advantage of FBA is that it allows an investigation of the desired organism without prior knowledge of enzyme kinetics. FBA is solely based on reaction stoichiometries and assumes an internal steady state for all metabolites, while allowing the uptake and production of compounds. Since kinetic information is most often limiting, this approach has proven useful for many larger scale networks but can also be applied readily to small sub-networks covering the most important constraints of the system.

\section{Modeling techniques}

The general formulation of the linear problem solved in FBA is:

$$
\begin{aligned}
& \text { Optimize } Z \\
& \text { s.t. } \\
& N \cdot \vec{v}=0 \\
& a_{j} \leq v_{j} \leq b_{j}
\end{aligned}
$$

where $N$ is the stoichiometric matrix of all reactions and $v$ is the flux vector representing the reaction fluxes. $a_{j}$ and $b_{j}$ are the lower and upper bounds for each flux $v_{j}$ in $v$. For irreversible reactions, $a_{j}$ is set to 0 , while as default no other bounds are applied. We performed several scans to investigate maintenance energy requirements and redox state. These scans are based on the common FBA formulation. Scanning is then performed by adding a constraint $\left(a_{j}=b_{j}\right)$ in Eq. (3) or by adding a sum constraint to address questions like ATP per biomass carbon. For ATP per biomass carbon, the constraint would look like:

$$
c \cdot v_{\text {Biomass }}-v_{\text {ATP consumption }}=0
$$

where $c$ would be the amount of ATP per produced biomass. To reduce the influence of possible alternate optima, each optimal solution was then flux minimized. This is achieved by splitting all reactions into forward and backward reactions, and minimizing the total flux in the network. To retain the detected optima (e.g., maximal biomass), the biomass flux was fixed at the optimal value during minimization. All modeling was performed using ScrumPy. CPLEX was used as solver for FBA problems.
The model and the source code for model analysis is available at https://github.com/QTB-HHU/Paenibacillus. A list of all the reactions and accession numbers for all the enzymes carrying out these reactions is provided as Additional file 2.

\section{Additional files}

Additional file 1: Additional figures and tables

Additional file 2: List of all reactions contained in the network and the corresponding enzyme accession numbers.

\section{Abbreviations}

FBA: flux balance analysis; RAST: rapid annotation using subsystem technology; BDO: 2,3-butanediol; PTA: phosphotransacetylase; ACK: acetate kinase; ALDC: acetolactate decarboxylase; ATOAD: acetoacetate CoA transferase; AADC: acetoacetate decarboxylase; ALS: acetolactate synthase; ADH: acetaldehyde dehydrogenase; ALD: aldehyde/alcohol dehydrogenase; FHL: formatehydrogen lyase; $\mathrm{BDH}$ : 2,3-butanediol dehydrogenase; NAR: nitrate reductase; NIR: nitrite reductase; ATOB: acetyl-Coa acetyltransferase.

\section{Authors' contributions}

SSY and OE conceived and designed the study. NA performed the experiments on P. Polymyxa. TP and NA created the initial network reconstruction. TP refined the reconstruction and performed the analysis of the metabolic model. SSY, OE, NA and TP analyzed the data. SY and OE contributed reagents/materials/analysis tools. All authors contributed to writing the manuscript and interpreting the results. All authors read and approved the final manuscript.

\section{Author details}

${ }^{1}$ Synthetic Biology and Biofuels Group, International Centre for Genetic Engineering and Biotechnology, Aruna Asaf Ali Marg, New Delhi, India. ${ }^{2}$ Institute of Complex Systems and Mathematical Biology, University of Aberdeen, Aberdeen, UK. ${ }^{3}$ Life Sciences Research Unit, University of Luxembourg, Luxembourg City, Luxembourg. ${ }^{4}$ Cluster of Excellence on Plant Sciences (CEPLAS), Heinrich-Heine-University, Universitätsstraße 1, 40225 Düsseldorf, Germany.

\section{Acknowledgements}

Authors acknowledge financial support from Department of Biotechnology (DBT), Ministry of Science and Technology, Govt of India through Grant Reference No. BT/PB/Center/03/2011 and Biotechnology and Biological Sciences Research Council (BBSRC) through Grant Reference No. BB/J019712/1.

\section{Compliance with ethical guidelines}

Competing interests

The authors declare that they have no competing interests.

Received: 23 June 2015 Accepted: 9 September 2015

Published online: 25 September 2015

\section{References}

1. Anand R, Grayston S, Chanway C. N2-fixation and seedling growth promotion of lodgepole pine by endophytic Paenibacillus polymyxa. Microb Ecol. 2013;66:369-74.

2. Da Mota FF, Gomes EA, Seldin L. Auxin production and detection of the gene coding for the Auxin Efflux Carrier (AEC) protein in Paenibacillus polymyxa. J Microbiol. 2008:46:257-64.

3. Bashir Z, Kondapalii VK, Adlakha N, Sharma A, Bhatnagar RK, Chandel G, et al. Diversity and functional significance of cellulolytic microbes living in termite, pill-bug and stem-borer guts. Sci Rep. 2013;3:2558. 
4. Hassler T, Schieder D, Pfaller R, Faulstich M, Sieber V. Enhanced fed-batch fermentation of 2,3-butanediol by Paenibacillus polymyxa DSM 365. Bioresour Technol. 2012;124:237-44.

5. Edwards JS, Palsson BO. The Escherichia coli MG1655 in silico metabolic genotype: its definition, characteristics, and capabilities. Proc Natl Acad Sci USA. 2000;97:5528-33.

6. Orth JD, Conrad TM, Na J, Lerman JA, Nam H, Feist AM, Palsson BØ. A comprehensive genome-scale reconstruction of Escherichia coli metabolism-2011. Mol syst boil. 2011;7:535.

7. Duarte NC, Herrgard MJ, Palsson BO. Reconstruction and validation of Saccharomyces cerevisiae iND750, a fully compartmentalized genomescale metabolic model. Genome Res. 2004;14:1298-309.

8. Mo ML, Palsson BO, Herrgard MJ. Connecting extracellular metabolomic measurements to intracellular flux states in yeast. BMC syst boil. 2009;3:37.

9. Marwoto B, Nakashimada Y, Kakizono T, Nishio N. Metabolic analysis of acetate accumulation during xylose consumption by Paenibacillus polymyxa. Appl Microbiol Biotechnol. 2004;64:112-9.

10. Gupta A, Murarka A, Campbell P, Gonzalez R. Anaerobic Fermentation of Glycerol in Paenibacillus macerans: metabolic pathways and environmental determinants. Appl Environ Microbiol. 2009;75:5871-83.

11. Adlakha N, Kushwaha HR, Rajagopal R, Yazdani SS. Draft genome sequence of the Paenibacillus sp. strain ICGEB2008 (MTCC 5639) isolated from the gut of Helicoverpa armigera. Genome Announc. 2013; doi:10.1128/genomeA.00026-12.

12. Adlakha N, Rajagopal R, Kumar S, Reddy VS, Yazdani SS. Synthesis and characterization of chimeric proteins based on cellulase and xylanase from an insect gut bacterium. Appl Environ Microbiol. 2011;77:4859-66.

13. Adlakha N, Sawant S, Anil A, Lali A, Yazdani SS. Specific fusion of beta1,4-endoglucanase and beta-1,4-glucosidase enhances cellulolytic activity and helps in channeling of intermediates. Appl Environ Microbiol. 2012;78:7447-54.

14. Adlakha $N$, Yazdani SS. Efficient production of ( $R, R$ )-2,3-butanediol from cellulosic hydrolysate using Paenibacillus polymyxa ICGEB2008. J Ind Microbiol Biotechnol. 2015:42:21-8.

15. Kauffman KJ, Prakash P, Edwards JS. Advances in flux balance analysis. Curr Opin Biotechnol. 2003;14:491-6.

16. Pfau T, Christian N, Ebenhoh O. Systems approaches to modelling pathways and networks. Brief Funct Genomics. 2011;10:266-79.

17. Zeng AP, Sabra W. Microbial production of diols as platform chemicals: recent progresses. Curr Opin Biotechnol. 2011;22:749-57.

18. Pokusaeva K, Fitzgerald GF, Sinderen D. Carbohydrate metabolism in Bifidobacteria. Genes Nutr. 2011;6:285-306.
19. Bianchetti CM, Elsen NL, Fox BG, Phillips GN. Structure of cellobiose phosphorylase from Clostridium thermocellum in complex with phosphate. Acta Crystallogr Sect F Struct Biol Cryst Commun. 2011;67:1345-9.

20. Caspi R, Altman T, Billington R, Dreher K, Foerster H, Fulcher CA, Holland TA, et al. The MetaCyc database of metabolic pathways and enzymes and the BioCyc collection of pathway/genome databases. Nucleic Acids Res. 2010;38:473-9.

21. Wiesenborn DP, Rudolph FB, Papoutsakis ET. Coenzyme A Transferase from Clostridium acetobutylicum ATCC 824 and its role in the uptake of acidsAppl. Environ Microbiol. 1989;55:323-9.

22. Lal S, Romano S, Chiarini L, Signorini A, Tabacchioni S. The Paenibacillus polymyxa species is abundant among hydrogen-producing facultative anaerobic bacteria in Lake Averno sediment. Arch Microbiol. 2012;194:345-51.

23. Yamanaka T, Ota A, Okunuki K. Oxidative phosphorylation coupled with nitrate respiration. I. Evidence for phosphorylation coupled with nitrate reduction in a cell-free extract of Pseudomonas aeruginosa. J Biochem. 1962;51:253-8.

24. Schepers HJ, Bringermeyer S, Sahm H. Fermentation of $\mathrm{D}$-xylose to ethanol by Bacillus macerans. Z Naturforsch. 1987:42:401-7.

25. Munjal N, Mattam AJ, Pramanik D, Srivastava PS, Yazdani SS. Modulation of endogenous pathways enhances bioethanol yield and productivity in Escherichia coli. Microb Cell Fact. 2012;11:145.

26. Sriram G, Gonzalez-Rivera O, Shanks JV. Determination of biomass composition of Catharanthus roseus hairy roots for metabolic flux analysis. Biotechnol Prog. 2006;22:1659-63.

27. Hara A, Radin NS. Lipid extraction of tissues with a low-toxicity solvent. Anal Biochem. 1978;90:420-6.

28. Aziz RK, Bartels D, Best AA, DeJongh M, Disz T, Edwards RA, Formsma K, et al. The RAST server: rapid annotations using subsystems technology. BMC Genom. 2008:9:75.

29. Russell JA. The colorimetric estimation of reaction by the Phenol-Hypochlorite of small amounts of ammonia. J Biol Chem. 1944;156:457-62.

30. Middleton KR. The use of the orange I method for determining soil nitrates and a comparison with the phenol-sulphonic acid method for certain soils of Northern Nigeria. J Sci Food Agric. 1959;10:218-24.

31. Tannock GW. Identification of Lactobacilli and Bifidobacteria. Curr Issues Mol Biol. 1999;1:53-64.

32. Poolman MG. ScrumPy: metabolic modelling with Python. Syst Biol. 2006;153:375-8

\section{Submit your next manuscript to BioMed Central and take full advantage of:}

- Convenient online submission

- Thorough peer review

- No space constraints or color figure charges

- Immediate publication on acceptance

- Inclusion in PubMed, CAS, Scopus and Google Scholar

- Research which is freely available for redistribution

Submit your manuscript at

www.biomedcentral.com/submit

C Biomed Central 